\title{
Search for weak neutrino point sources using angular auto-correlation analyses in IceCube
}

\author{
The IceCube Collaboration ${ }^{\dagger}$ \\ $\dagger$ http://icecube.wisc.edu/collaboration/authors/icrc17_icecube \\ E-mail: tglauch@icecube.wisc.edu, aturcati@icecube.wisc.edu
}

\begin{abstract}
The IceCube Neutrino Observatory is a cubic kilometer neutrino telescope located in the Antarctic ice. Recently, IceCube has measured a diffuse all-sky all-flavor astrophysical neutrino flux above $30 \mathrm{TeV}$. However, the origin and production of these particles still remain unknown. Given the observed flux, the absence of observations of bright point-sources could be explained by a large population of weak sources. This scenario can be tested using autocorrelation methods. We present here the results of two independent analyses on two different neutrino samples based on 6 and 7 years of IceCube data. The first sample is optimized for events coming from the Northern Hemisphere, while the second one is an all-sky sample. Experimental results are obtained by analyzing these samples using a multipole expansion and a two-point angular correlation technique, respectively. In addition, flux upper limits for extended sources and the Cygnus region have been calculated.
\end{abstract}

Corresponding authors: Theo Glauch ${ }^{1,2}$, Andrea Turcati ${ }^{2}$, Kai Krings ${ }^{2 *}$

${ }^{1}$ III. Physikalisches Institut, RWTH Aachen University, D-52056 Aachen, Germany

2 Physik-department, Technische Universität München, D-85748 Garching, Germany

35th International Cosmic Ray Conference - ICRC2017

10-20 July, 2017

Bexco, Busan, Korea

${ }^{*}$ Speaker. 


\section{Introduction}

The origin of charged cosmic rays (CRs), as well as their production mechanisms are one of the most important questions in modern astroparticle physics. In fact, there are reasons to believe that the production of CRs and neutrinos are closely connected. In source environments where protons are accelerated and undergo proton-proton or photonuclear interactions, a cascade of light mesons evolves which subsequently decay into gamma-rays, leptons and (highly-energetic) neutrinos [1]. The latter are the ideal tracer for these processes since they are neither attenuated nor deflected during their travel through the universe and hence point back to their origin. In recent years the multi-messenger approach has been used to derive upper flux limits for various classes of neutrino point-sources [2]. These limits suggest that the observed neutrino flux may originate from the superposition of many weak sources of various types. This motivates our angular correlation approach, searching for a small-scale clustering in the astrophysical neutrino flux coming from a large population of weak neutrino point-sources.

IceCube is a neutrino detector designed for the measurement of highly-energetic, i.e. astrophysical neutrinos. The cubic-kilometer detector is installed in the ice at the geographic South Pole between depths of $1450 \mathrm{~m}$ and $2450 \mathrm{~m}$ [3]. Detector construction started in 2005 and finished in 2010. Neutrino reconstruction relies on the optical detection of Cherenkov radiation emitted by secondary particles produced in neutrino interactions in the surrounding ice or the nearby bedrock $[4,5]$. At energies $E_{v}>100 \mathrm{TeV}$ the mean opening angle between the direction of a secondary muon and the direction of its primary muon-neutrino is less then $0.03^{\circ}$ [6]. This motivates the use of through-going muons, which have a median angular reconstruction uncertainty better than $1^{\circ}$ at energies above $10 \mathrm{TeV}$, to search for point-sources [7, 8].

The two analyses presented in this work make use of the direction and energy information of through-going muon measured by the IceCube detector and collected in two different samples. The Multipole Analysis is applied to a data sample of through-going muons with declination $\delta>-5^{\circ}$. The sample was originally developed for the characterization of the diffuse astrophysical neutrino flux in [7]. However, due to its good background rejection (neutrino purity of 99.7\%) and good statistics (around 500 astrophysical neutrinos in six years of data) it is also well suited for pointsource searches. The 2pt-Autocorrelation Analysis is instead applied to an all-sky data sample, consisting of through-going muons coming from both hemispheres during an integrated livetime of seven years. This sample is also used in the Single Point-Source Analysis that provides the best limits so far available [8]. Although mathematically very similar the two analyses follow a different philosophy in their implementations. While the 2pt-Autocorrelation analyses is almost model independent, the Multipole Analysis uses the fitted astrophysical neutrino spectrum from [7] to introduce an optimal energy weighting for the astrophysical flux. The two analyses presented in this paper are closely related to the already published Hotspot Population Search [8] which also searches for a population of weak neutrino point-sources. But while the latter analysis counts the number of neutrino hotspots above a certain flux level, the analyses presented here make use of the angular clustering of the neutrino events, making them more powerful for a high number of sources (also compare results in Figure 2). Stacking Analyses, another extension of point-source analysis only search for a time-integrated flux from a source catalog, while our approach is more generally searching for clustering anywhere in the sky. 


\section{Methods}

As stated before, the analyses presented in this paper search for a large population of weak point-sources as opposed to a purely isotropic flux. This can be precisely stated as the following two hypotheses:

- Background-Hypothesis: The neutrino flux measured by IceCube consists exclusively of atmospheric neutrinos and an isotropic astrophysical neutrino component.

- Signal-Hypothesis: The neutrino flux measured by IceCube consists of an atmospheric and an astrophysical neutrino component. A fraction of the observed diffuse astrophysical flux is clustered in isotropically distributed, equally strong neutrino point-sources.

Apart from the analysis of the Cygnus region, we will always refer to these definitions.

\subsection{Multipole}

The Multipole Analysis is one possibility to calculate the two-point autocorrelation of the neutrino flux on a specific angular scale $\alpha$. It is based on the expansion of the neutrino skymap into spherical harmonics $Y_{\ell}^{m}(\theta, \varphi)$ according to

$$
f(\theta, \varphi)=\sum_{\ell=0}^{\infty} \sum_{m=-\ell}^{\ell} a_{\ell}^{m} Y_{\ell}^{m}(\theta, \varphi),
$$

with zenith angle $\theta$ and azimuth angle $\varphi$. The $a_{\ell}^{m}$ coefficients describe structures with an angular size of $\alpha \sim 180 / \ell$. In order to increase the analysis' sensitivity, neutrino events are not equally weighted, but injected in skymaps with their probability $\mathscr{P}($ astro $\mid E)$ of being of astrophysical origin

$$
\mathscr{P}(\text { astro } \mid E)=\frac{1}{1+\frac{\mathscr{E}_{B}(E)}{\mathscr{E}_{S}(E)} \cdot \frac{n_{\text {tot }}-n_{\text {astro }}}{n_{\text {astro }}}},
$$

with a-priori energy distributions $\mathscr{E}_{B}(E)$ and $\mathscr{E}_{S}(E)$ for atmospheric and astrophysical neutrinos, respectively. $n_{\text {tot }}$ and $n_{\text {astro }}$ are the total number of neutrinos and the number of astrophysical neutrinos in the sample, respectively.

In order to quantify the correlation of neutrino events the angular power spectrum $C_{\ell}$ is used

$$
C_{\ell}=\frac{1}{2 \ell+1} \sum_{m=-\ell}^{m=\ell}\left|a_{\ell}^{m}\right|^{2}
$$

In [9], it is shown that this definition gives an unbiased estimator for the two-point autocorrelation of a signal on a sphere. For this work, however, we omit the $m=0$ coefficients, since they only include zenith information and therefore coincide with the direction of IceCube's largest detector systematics. Furthermore the angular power spectrum is normalized in order to avoid fake sensitivities caused by fluctuations of the baseline value, which arise when injecting independent Monte Carlo events. Hence, for the following we use the modified angular power spectrum

$$
C_{\ell}^{\mathrm{eff}} \rightarrow \frac{C_{\ell}^{\mathrm{eff}}}{\sum_{\ell=0}^{\ell_{\max }} C_{\ell}^{\mathrm{eff}}} .
$$


Using Monte Carlo generated skymaps the probability density function for each $C_{\ell}^{\text {eff }}$ can be shown to be nearly Gaussian in the region of interest. Hence a $\chi^{2}$-inspired test-statistic is used to distinguish background and signal hypothesis

$$
T S=\frac{1}{\sum_{\ell} w_{\ell}^{\mathrm{eff}}} \cdot \sum_{\ell=1}^{\ell_{\max }} w_{\ell}^{\mathrm{eff}} \operatorname{sign}_{\ell}\left(\frac{C_{\ell}^{\mathrm{eff}}-\left\langle C_{\ell, \mathrm{bg}}^{\mathrm{eff}}\right\rangle}{\sigma_{\ell, \mathrm{bg}}}\right)^{2}
$$

Here, $\left\langle C_{\ell, \mathrm{bg}}^{\text {eff }}\right\rangle$ is the background median with standard deviation $\sigma_{\ell, \mathrm{bg}}$. The angular weights $w_{\ell}^{\text {eff }}$ are a function of $\ell$ complying with the fact that point-sources are expected to have a typical angular size of around $1^{\circ}$ corresponding to the angular reconstruction error of the neutrino events. The weights are deduced from 10'000 Monte Carlo generated skymaps with all astrophysical events clustered in point-sources [10]. Using the median of the resulting signal angular power spectrum $\left\langle C_{\ell, \text { sig }}\right\rangle$ the weights are given by

$$
w_{l}^{\mathrm{eff}}=\frac{\left\langle C_{\ell, \mathrm{sig}}^{\mathrm{eff}}\right\rangle-\left\langle C_{\ell, \mathrm{bg}}^{\mathrm{eff}}\right\rangle}{\sigma_{\ell, \mathrm{bg}}} .
$$

Finally, the signum function in equation (2.5) ensures that only deviations in the expected direction contribute a positive value to the test-statistic value. Monte Carlo studies show that the test-statistic distributions for all signal strength are well described by a convolution of a Gaussian with an exponential function.

\subsection{2pt-Autocorrelation}

Autocorrelation tests making use of two-point correlation functions are solid statistical methods to evaluate the amount of spatial clustering over different angular scales of a set of events distributed on a skymap. An analysis using a similar method has been performed over earlier versions of IceCube data [11]. The test described here makes use of the distribution of the angular distances between event-pairs $(i, j)$ to distinguish a potential clustered signal, from the isotropic background hypothesis. The test has been developed to be almost completely data-driven, in the sense that no prior assumptions on the signal model are needed to perform the analysis. The autocorrelation function is defined as the cumulative number of event-pairs having an angular distance $\Psi_{i, j}$ minor or equal a given angular distance $\alpha$,

$$
N(\alpha)=\sum_{i, j>i} \Theta\left(\alpha-\Psi_{i, j}\right)
$$

where $\Theta$ is the Heaviside step function. The calculation is then repeated in a range of $\alpha$ that goes from $0.25^{\circ}$ to $5^{\circ}$ in steps of $\Delta \alpha=0.25^{\circ}\left(\Delta \alpha=0.1^{\circ}\right.$ for the analysis in the Cygnus Region).

Since astrophysical neutrinos are often expected to have a harder spectrum compared to the atmospheric neutrino background, it is possible to enhance the sensitivity of the analysis by introducing an appropriate weighting system. In a similar way of what is shown in [12], a weighting scheme proportional to the energies $\left(E_{i}, E_{j}\right)$ of the neutrinos belonging to each event-pair has been chosen. The weighted cumulative number of pairs takes the following form,

$$
N(\alpha)=\sum_{i, j>i} w_{i} \cdot w_{j} \cdot \Theta\left(\alpha-\Psi_{i, j}\right),
$$


where $w_{i}, w_{j}$ are the weights assigned to each event. Accordingly to the data-driven purposes of the method, the weights are derived from the normalized energy distribution $\mathscr{P}(E)$ of the data on the skymap, independent from specific signal models. Each weight is proportional to the logarithm of the probability of observing an event with equal or higher energy in the sample and it is calculated as

$$
w_{i}=w\left(E_{i}\right)=-\log _{10}\left(1-\int_{0}^{E_{i}} \mathscr{P}(E) \mathrm{d} E\right) .
$$

For each angular scale $\alpha$, the background distribution is derived by scrambling the data in right ascension to obtain isotropic skymaps. The distribution is then fitted with a Gamma function, that is used to calculate the p-value $(p(\alpha))$ of a given skymap realization. The lowest $\mathrm{p}$-value from the different angular scales is then selected to define the test statistic,

$$
T S=-\log _{10}\left(p(\alpha)_{\text {Best }}\right) .
$$

As the last step, to correct for the trial factor due to the binning of the angular scales, a large number of realizations of isotropic skymaps has to be analyzed, in order to obtain the $T S$ p.d.f. The trial corrected p-value then corresponds to the probability of obtaining a $T S$ at least as large as the one obtained from data.

\section{Results}

\subsection{North}

The left panel of Figure 1 shows the results of the 2pt-Autocorrelation analysis in the Northern Hemisphere. The trial corrected p-value is $46 \%$, consistent with fluctuations from background expectations. The right panel of the figure shows the angular power spectrum of the neutrino flux from the Northern Hemisphere in black dots. The blue solid and shaded area indicate the median and standard deviation from simulated Monte Carlo skymaps, respectively. The pulls are shown in the bottom panel. There is no obvious structure visible. The test-statistic value is calculated from equation (2.5) and compared to the expectation for the background hypothesis giving a pvalue of $31.5 \%$. The non-observation of point-source clustering is used to calculate Neyman upper limits on the flux-per-source for the model of $N_{\text {Sou }}$ equally strong point-sources. The results are shown in Figure 2 for an astrophysical power-law with spectral index $\gamma=2.0$. The intersection of the diffuse flux and the $90 \%$ upper limit on the point-source is at around 500 sources with $E_{v}^{2} \partial \Phi / \partial E_{v} \leq 10^{-13} \mathrm{TeV} \mathrm{cm}^{-2} \mathrm{~s}^{-1}$. In addition to the two analyses presented here, refined upper limit fluxes for the Hotspot Population Analysis are also shown in Figure 2 [8] for comparison.

Considering the various results, there is increasing evidence that the observed flux of astrophysical neutrinos is not produced by a few predominant point-sources in the neutrino sky. Anyway, the results are consistent with the point-source scenario in general. As discussed in the introduction there are many source types that are too abundant to be visible in our analyses. There are, for example, $\mathscr{O}\left(10^{8}\right)$ Starburst Galaxies in the observable universe, while the presented analyses are only sensitive up to the crossing point of diffuse flux and sensitivity line at $\sim 700$ sources. One approach to trace such abundant source classes is to include other messenger-particles as cosmicrays or various kinds of electromagnetic radiation to get a complete view on the origin of cosmic radiation. 

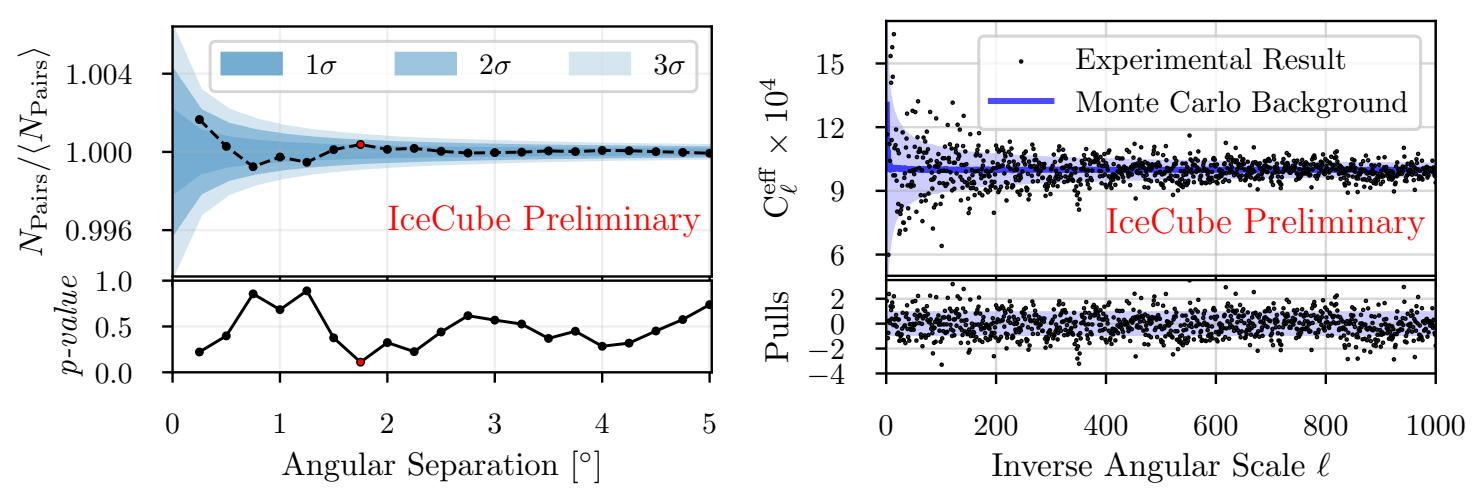

Figure 1: Left (2pt-Autocorrelation): In the top panel is shown the experimental number of pairs divided by the mean number of pairs expected from a pure background hypothesis as a function of the angular scale $\alpha$, the shaded areas represent three different sigma bands. The lower panel shows the pre-trial p-value corresponding to each dot. The best clustering scale is marked with a red dot. Right (Multipole): In the top panel the experimental angular power spectrum (black dots) is shown in comparison with the Monte Carlo background expectation as a function of $\ell$. The corresponding pulls, i.e. the deviation in Gaussian sigma is shown in the bottom panel.

\subsection{South}

Similar to what has been done for the Northern Hemisphere, the Southern Hemisphere is also studied using the 2pt-Autocorrelation method and yielding a non-observation of any clustering, and hence upper limits are calculated. The left panel of Figure 3 shows the results of the 2ptAutocorrelation analysis in the Southern Hemisphere. The trial corrected p-value is $60 \%$, which is again consistent with fluctuations from background expectations.

\subsection{Cygnus}

The Cygnus Region is a Galactic region here formally defined as the area of the sky extending between $72^{\circ}$ and $83^{\circ}$ in galactic longitude and $-3^{\circ}$ and $4^{\circ}$ in galactic latitude. It has been intensively studied by $\gamma$-ray experiments in the $\mathrm{GeV}$ and $\mathrm{TeV}$ energy ranges [13]. This region has also been considered as a possible location for CR acceleration and neutrino production [14, 15]. For these reasons, the 2pt-Autocorrelation method has been applied on the identified region, considering two different scenarios: point-like sources, as done for the two hemispheres as described above, and extended sources with a Gaussian shape. The right panel of Figure 3 shows the results of the 2 pt-Autocorrelation analysis. The trial corrected p-value is 53\%, again consistent with fluctuations from background expectations. 


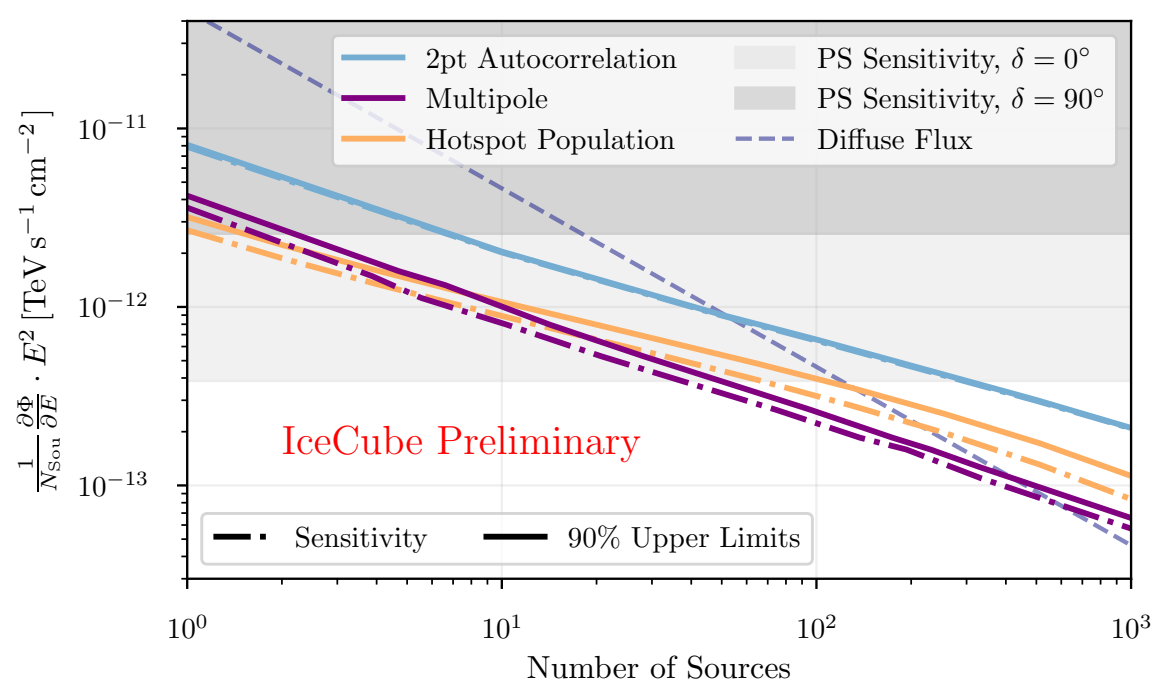

Figure 2: The plot shows the Northern Hemisphere's 90\% C.L. flux upper limits for equally strong point-sources and draws a comparison to refined results of [8]. The dashed, blue line shows the diffuse astrophysical neutrino flux distributed equally over the number of sources. The gray shaded areas show the sensitivity of the single point-source analysis as in [8]. It can be seen that for a large amount of sources contributing, the flux limits per source of the angular autocorrelation analyses drop under the point-source sensitivity line. At an even higher number of sources, behind the intersection point, these limits are dominated by the constraints from the diffuse astrophysical flux.
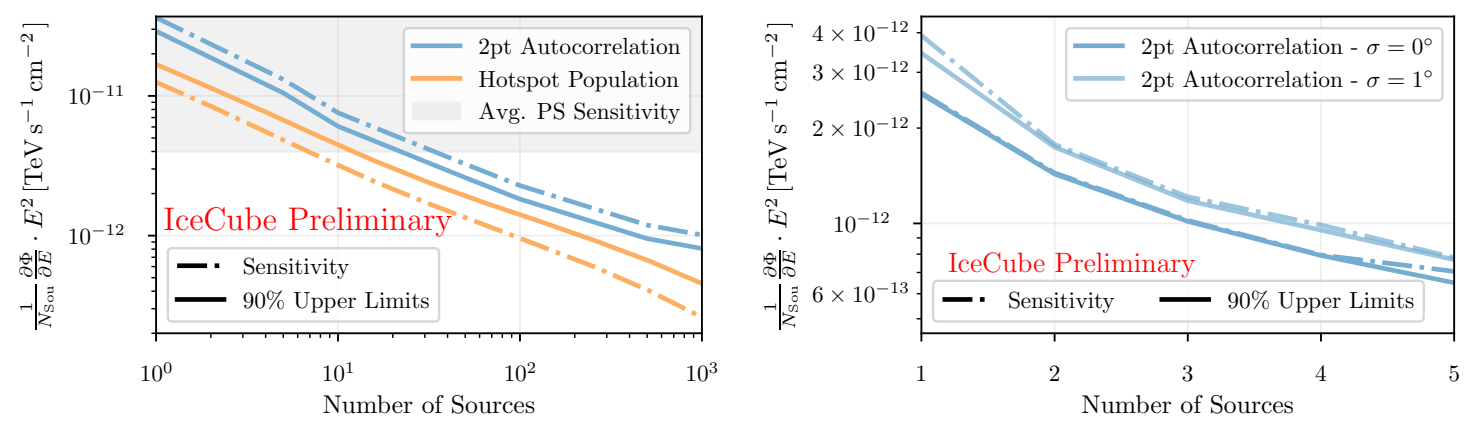

Figure 3: Left: Southern Hemisphere. The plot shows the 90\% C.L. flux upper limits of the 2pt Autocorrelation analysis and draws a comparison to refined results of a similar analysis described in [8]. The gray shaded area show the average sensitivity of the Single Point-Source Analysis as in [8]. It can be seen that for a large amount of sources contributing, the flux limits per source of the analyses drop under the point-source sensitivity line. Right: Cygnus Region. The plot shows the $90 \%$ C.L. flux upper limits of the 2pt Autocorrelation analysis for point-like $\left(\sigma=0^{\circ}\right)$ and extended, Gaussian shaped sources $\left(\sigma=1^{\circ}\right)$. 


\section{Conclusions}

Two different and complementary analyses focused on the observation of clustering of neutrino events have been presented. The two analyses have been performed on two different neutrino samples with statistics exceeding six years of detector livetime. The Northern Hemisphere, the Southern Hemisphere and the Cygnus Region have been studied separately and the results of the observations are compatible with background expectations. On this basis upper limits have been calculated for all the scenarios, among which the strongest neutrino flux upper limits come from the northern hemisphere.

\section{References}

[1] S. R. Kelner, F. A. Aharonian, and V. V. Bugayov, Phys. Rev. D 74 (Aug, 2006) 034018.

[2] M. Kowalski, J. Phys. Conf. Ser. 632 (2015) 012039.

[3] IceCube Collaboration, M. G. Aartsen et al., Journal of Instrumentation 12 (2017) P03012.

[4] IceCube Collaboration, M. G. Aartsen et al., Journal of Instrumentation 9 (2014) P03009.

[5] AMANDA Collaboration, J. Ahrens et al., Nucl. Instrum. Meth. A524 (2004) 169-194.

[6] J. G. Learned and K. Mannheim, Annual Review of Nuclear and Particle Science 50 (2000) 679-749.

[7] IceCube Collaboration, M. G. Aartsen et al., The Astrophysical Journal 833 (2016) 3.

[8] IceCube Collaboration, M. G. Aartsen et al., The Astrophysical Journal 835 (2017) 151.

[9] C. Copi, D. Huterer, D. Schwarz, and G. Starkman, Phys. Rev. D75 (2007) 023507.

[10] IceCube Collaboration, M. G. Aartsen et al., Astropart. Phys. 66 (2015) 39-52.

[11] IceCube Collaboration, M. G. Aartsen et al., Astropart. Phys. 66 (2015) 39-52.

[12] IceCube Collaboration, A. Bernhard and S. Odrowski, , in Proceedings, 33rd International Cosmic Ray Conference (ICRC2013): Rio de Janeiro, Brazil, July 2-9, 2013, p. 0471.

[13] A. A. Abdo et al., Astrophys. J. 753 (2012) 159.

[14] T. M. Yoast-Hull, J. S. Gallagher III, F. Halzen, A. Kheirandish, and E. G. Zweibel, arXiv: 1703.02590.

[15] M. Guenduez, J. Becker Tjus, B. Eichmann, and F. Halzen, arXiv:1705.08337. 